\title{
The Future of the Internet of Things
}

\author{
Thamer Al-Rousan
}

\begin{abstract}
The significance of the Internet of Things (IoT) in current trends is continuously rising. IoT is a concept that encompasses various objects and methods of communication to exchange information. Today IoT is more a descriptive term of a vision that everything should be connected to the internet. IoT will be fundamental in the future because the concept opens up opportunities for new services and new innovations. The applications of the IoT are varied and numerous; they range from relatively simple home automation scenarios to the much more complex scenarios of interconnected smart cities. IoT is expected to dominate the future with huge amounts of content oriented traffic that is a result of intensive interactions between the millions of devices that will be available by then. The rising popularity of IoT has been accompanied by a corresponding rise in the number of challenges. In this paper we focus on the current state of IoT, possible usage scenarios and challenges that influence the adoption of the Internet of Things.
\end{abstract}

Keywords - Internet of Things, smart things.

\section{INTRODUCTION}

The Internet is a powerful global communication medium that provides instantaneous information across geographical, cultural, language, and time spheres [1]. Internet is a network of networks that consists of millions of private, public, academic, research, business, and government networks, of local to global scope, that are linked by a broad array of electronic, wireless and wired networking technologies [2]. The internet brought marvelous changes into our daily life without leaving any field like day to day personal work, health, education, research, humanity, education, tourism, science, entertainment, government sectors, business, communication, service, manufacturing and so on. More than 180 countries are linked into exchanges of data, information, news and opinions. According to Internet World statistics, there was an estimated of 326, 72, 33, 742 Internet users at global level. This represents almost $42.7 \%$ of the total world's population.

The next wave in the area of Internet will be Internet of Things. In the Internet of Things (IoT) paradigm, many of the things that surround us will be on the network in one form or another.

\footnotetext{
Faculty of Science and Information Technology
Isra University, (E-mail: thamer.rousan@iu.edu.jo)
}

Thing can be defined as an entity, an idea, a quality perceived, or thought to have its own existence in the world. When we are talking about things, they could be both Living Things and Non-Living Things. Things, in this context, can be people, animals, plants, birds, servers, applications, shampoo bottles, cars, steering wheels, coffee machines, electronic devices, park benches or just about any other random item that comes to our mind, even which could be vicinity dust also. Everyday objects include not only electronic devices we encounter but also use daily, and technologically advanced products such as equipment and electronic gadgets, but "things" that we do not do normally think of as electronic at all - such as food, clothing, and furniture, materials, parts, merchandise and specialized items, landmarks, monuments and works of art and all the miscellany of commerce, culture and sophistication [3]. Once something has a unique identifier, it can be tagged, assigned a Uniform Resource Identifier (URI) and monitored over a network, automated other things and even talk too.

\section{INTERNET OF THINGS}

The Internet of Things is an important topic in technology industry, policy, and engineering circles and has become headline news in both the specialty press and the popular media. This technology is embodied in a wide spectrum of networked products, systems, and sensors, which take advantage of advancements in computing power, electronics miniaturization, and network interconnections to offer new capabilities not previously possible [2]. An abundance of conferences, reports, and news articles discuss and debate the prospective impact of the "IoT revolution"-from new market opportunities and business models to concerns about security, privacy, and technical interoperability [4].

The large-scale implementation of IoT devices promises to transform many aspects of the way we live. For consumers, new IoT products like Internet-enabled appliances, home automation components, and energy management devices are moving us toward a vision of the "smart home", offering more security and energy- efficiency [5]. Other personal IoT devices like wearable fitness and health monitoring devices and network- enabled medical devices are transforming the way healthcare services are delivered. This technology promises to be beneficial for people with disabilities and the elderly, enabling improved levels of independence and quality of life at a reasonable cost [2]. IoT systems like networked 
vehicles, intelligent traffic systems, and sensors embedded in roads and bridges move us closer to the idea of "smart cities", which help minimize congestion and energy consumption[6]. IoT technology offers the possibility to transform agriculture, industry, and energy production and distribution by increasing the availability of information along the value chain of production using networked sensors. However, IoT raises many issues and challenges that need to be considered and addressed in order for potential benefits to be realized [7].

A number of companies and research organizations have offered a wide range of projections about the potential impact of IoT on the Internet and the economy during the next five to ten years. Cisco, for example, projects more than 24 billion Internet-connected objects by 2019 [9]; Morgan Stanley, however, projects 75 billion networked devices by 2020 . Looking out further and raising the stakes higher, Huawei forecasts 100 billion IoT connections by 2025[10]. McKinsey Global Institute suggests that the financial impact of IoT on the 5 global economy may be as much as $\$ 3.9$ to $\$ 11.1$ trillion by 2025 [11]. While the variability in predictions makes any specific number questionable, collectively they paint a picture of significant growth and influence [12].

Some observers see the IoT as a revolutionary fullyinterconnected "Smart Thing" of progress, efficiency, and opportunity, with the potential for adding billions in value to industry and the global economy. Others warn that the IoT represents a darker world of surveillance, privacy and security violations, and consumer lock-in. In next subsection, this study will discuss the evolution and the concept of Smart Things and the challenges related to IoT.

\section{SMART THINGS}

Smart Things make our world smarter. Smart Things are a group of devices which can be monitored and controlled via a hub device (central processors) and web services. Smart Things adding support for popular connected products such as the Belkin WeMo family of devices, Philips Hue colorchanging bulbs, and the Sonos home music system. The idea of smart objects and the IoT was recently popularized [13]. As with those products, Smart Things users will now be able to control and automate today's additions directly through the Smart Things applications. The world of smart fridges, smart washing machines, smart TV, other home appliances, smart shoes and smart phones is already in use, but the practice of user experience design for Internet of Things is still fairly new concept [3]. Smart Things are one of the latest technology launched by innovators of Kickstarter. Design companies like IDEO and frog design are frequently asked to design products that unify software interaction, device design and service design - which are all the key components of Internet of Things. The current transition of the global Internet to IPv6 will provide a virtually unlimited number of public IP addresses able to provide bidirectional and symmetric (Machine-Machine: M2M) access to billions of Smart Things. Interaction and integration of Internet of Things in the global Internet are IPv6 integration, global interoperability, IoT - Cloud integration. In other words, how to bridge billion of Smart Things globally, while respecting their specific constraints [3]. Weaving Smart Things, enterprises, and people leads to innovation in products, services and new business models in the fourth coming years.

Smart Things are autonomous physical or digital objects augmented with sensing, processing, acting and network capabilities. Adding smartness to everyday things in our world, so that our life can be more amazing. Smart Things places the world of linked things at your fingertips. Smart Things are more intelligent, convenient, secure, safe and efficient. These make it easy to connect the things in our physical world to the internet for automate, monitor, control and have fun with them from anywhere, at any time through any network by any one. This smartness can be done by different embedded Internet of Things technologies like RFID, EPC, barcode, IPv4 / IPv6, sensors, actuators, GIS, GPS, WiFi, Bluetooth, ZigBee, NFC, ambient intelligence, Web 3.0 and telemedicine. We call smart any physical object connected to the web with some sensing capabilities. Its main capabilities are [14]:

1) Detect users and the social connections between them.

2) Access user's data.

3) Infer social context according to user's network topology, preferences and features

4) Infer social goals according to the social context and the user model

5) Coordinate their behavior 6) Provide a context driven output.

Smart Devices are characterized by the ability to execute multiple, possibly concurrent, applications, supporting different degree of mobility and customization and by supporting intermittent remote service and operating according to local resource constraints [15]. Smart Devices tend to be owned, operated, and configured and under the control of individual human users, For Example: personal computers, smart phones, cameras, game consoles, set up boxes and other computer peripherals, such as printers, mouse, external disk drives. In authors prediction IoT as a composition of smart objects that can understand and react to their environments. Based on practical experimentation and prototyping, smart objects are classified into Activity-aware objects, Policyaware objects and Process-aware objects [16]:

1) Activity-aware objects: Amount of work performed to convert input into output.

2) Policy-aware objects: The basic protocols issued by government or system administrator. 
3) Process-aware objects: A series of actions, motions, or occurrences.

They also identified the smart-object design space as a space of three dimensions: awareness, representation and interaction [3]. The Smart Things concept has four logical architectural layers[17]:

1) Which connect to the Smart Things Hub or in some cases directly to the Cloud.

2) Which acts as a gateway for getting events and messages to or from the Cloud.

3) Which provides the abstraction and intelligence layers described above, as well as the web services that support the presentation layer.

4) Which provides the presentation layer for smart things in the form of mobile applications and our web IDE.

Within the Smart Things-Cloud, however there are also four logical "Layers" of the architecture [18] as well:

1) Connectivity: Which is responsible for maintaining persistent connectivity to Smart Things Hubs, Smart Things Mobile application.

2) Event processing and routing: This layer routes events from hubs or devices to smart applications that are subscribed to specific devices or events.

3) Application: This layer provides the data access layer for data about accounts, users, and devices and is responsible for the execution of smart applications.

4) Web Services: This layer provides the web services or Application Programmatic Interface (API) layer that supports both the mobile applications as well as developers who want to integrate from an external system using the Smart Things APIs.

\section{A. Concepts}

One of the buzzwords in the arena of Internet of Things applications is Smart Things. These applications can be found in our daily life right from the morning wake up to till the late go to the bed [19]. Even in small villages, these applications are penetrated in the form of Smart Phones. Most of us think about "being connected" in terms of electronic devices such as servers, computers, tablets, telephones, smart phones and physical devices shoe, brush, bed, curtain and table. Smart Things describes a world where just about anything can be connected and communicates in an intelligent fashion that ever before. In other words, with the smart things, the physical world is becoming one big information system and Big Data Analytics (BDA) is helping in analysis for the better decisions.

Although the concept wasn't named until 1999, the Internet of Things has been in development for decades. The first Internet appliance was a coke machine at Carnegie Melon University in the early 1980s [7]. Programmers working several floors above the vending machine wrote a server program that tracked how long it had been since a storage column in the machine had been empty. The programmers could connect to the machine over the Internet, check the status of the machine and determine whether or not there would be a cold drink awaiting them, should they decide to make the trip down to the machine. Gradually importance of Smart Things under the umbrella of Internet of Things is grown up. There are various predictions about smart devices or things connecting to the internet based on different surveys. A report entitled 'Internet of Things [20] has predicted that there could be up to 16 billion connected devices by the year 2020 with an average of nearly 6 devices for every person on the planet. But IMS Research [17] forecasts that we will have 22 billion Internet connected devices by the year 2020 as shown in figure 1.

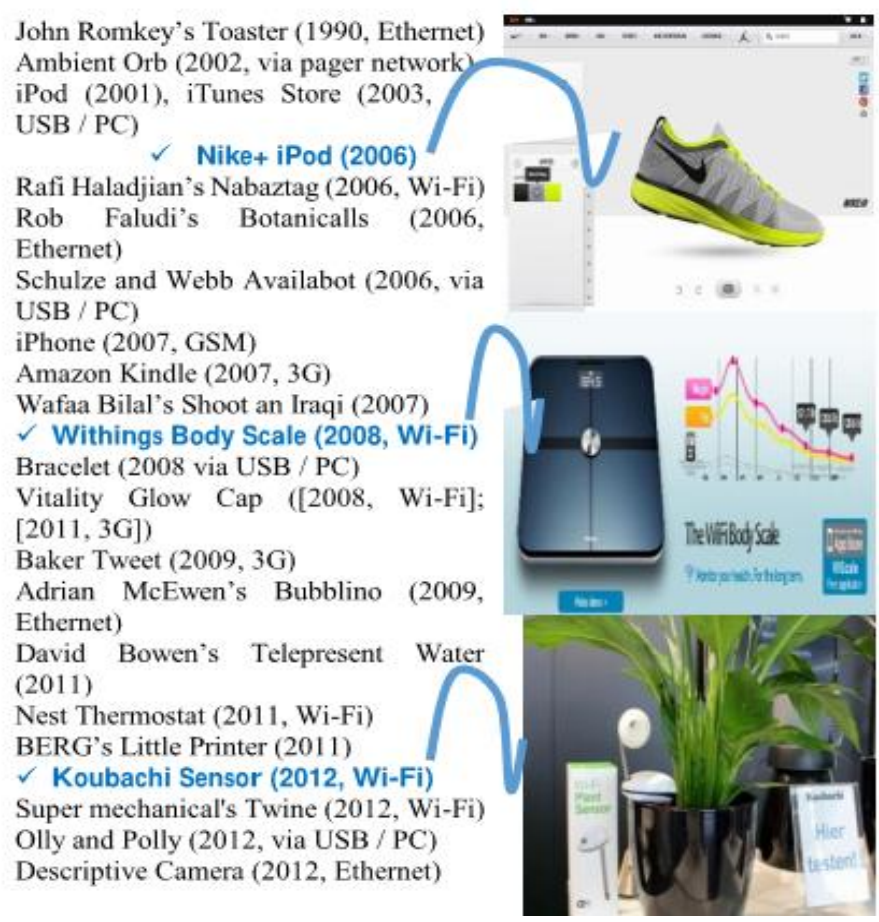

Fig. 1. Things connected through various IoT technologies.

\section{INTERNET OF THINGS CHALLENGES}

The Internet of Things raises significant challenges that could stand in the way of realizing its potential benefits. These challenges include [21], [22], [23], [24]: security; privacy; interoperability and standards; legal, regulatory, and rights; and emerging economies and development.

- Security: Many IoT implementations present new and unique security challenges. Addressing these challenges and ensuring security in IoT products and services must be a fundamental priority. Users need to trust that IoT devices and related data services are secure from vulnerabilities, especially as this technology become more pervasive and integrated into our daily lives. Poorly secured IoT devices and services can serve as potential entry points for cyber- 
attack and expose user data to theft by leaving data streams inadequately protected.

- Privacy: The full potential of the Internet of Things depends on strategies that respect individual privacy choices across a broad spectrum of expectations. The data streams and user specificity afforded by IoT devices can unlock incredible and unique value to IoT users, but concerns about privacy and potential harms might hold back full adoption of the Internet of Things. This means that privacy rights and respect for user privacy expectations are integral to ensuring user trust and confidence in the Internet, connected devices, and related services.

- Interoperability. Interoperability is defined as the ability of two or more systems to exchange information and also use the information that has been changed. Interoperability is important in the IoT as it will contain different heterogeneous objects, networks and thus systems that will need to work together to create an Internet of Things.

- Legal, Regulatory and Rights: The use of IoT devices raises many new regulatory and legal questions as well as amplifies existing legal issues around the Internet. The questions are wide in scope, and the rapid rate of change in IoT technology frequently outpaces the ability of the associated policy, legal, and regulatory structures to adapt.

- Emerging Economy and Development Issues: The Internet of Things holds significant promise for delivering social and economic benefits to emerging and developing economies. This includes areas such as sustainable agriculture, water quality and use, healthcare, industrialization, and environmental management, among others. As such, IoT holds promise as a tool in achieving the United Nations Sustainable Development Goals.

The broad scope of IoT challenges will not be unique to industrialized countries. Developing regions also will need to respond to realize the potential benefits of IoT. In addition, the unique needs and challenges of implementation in lessdeveloped regions will need to be addressed, including infrastructure readiness, market and investment incentives, technical skill requirements, and policy resources.

\section{CONCLUSION}

The Internet of Things is playing an active role in our everyday life, and its applications are fabulous and countless. Projections for the impact of IoT on the Internet and economy are impressive, with some anticipating as many as 100 billion connected IoT devices and a global economic impact of more than $\$ 11$ trillion by 2025 . The best part of Internet of Things is that they are bringing the quality of life to human beings, operational efficiency and handles the situations where human being intervention is not at all possible. At the same time, however, the Internet of Things raises significant challenges that could stand in the way of realizing its potential benefits.

In the future, we need to focus more on Internet of Things in terms of development, deployment, architectural, global level standardization, and ethical issues. We also need to concentrate on challenges associated with IoT in order for the potential benefits for individuals, society, and the economy to be realized.

\section{REFERENCES}

[1] S.-I. Hou, S.-A. R. Charlery, and K. Roberson, "Systematic literature review of Internet interventions across health behaviours," Health Psychology and Behavioral Medicine: Open Access Journal, vol. 2, no. 1, pp. 455-481, 2014.

[2] G. Nunberg, "The advent of the Internet," 2012.

[3] E. A. Kosmatos, N. D. Tselikas, and A. C. Boucouvalas, "Integrating RFIDs and smart objects into a unified Internet of things architecture,"

[4] Advances in Internet of Things, vol. 1, pp. 5-12, 2011.

[5] J. Antonio et al., "Drugs interaction checker based on IoT," Internet of Things (IOT), pp. 1-8, IEEE, 2010.

[6] T. Al-Rousan, "Cloud computing for global software development: Opportunities and challenges," in Transportation Systems and Engineering: Concepts, Methodologies, Tools, and Applications. IGI Global, 2015, pp. 897-908.

[7] E. Wasserman, "Riding herd: RFID tracks live-stock," 2009.

[8] E. Biddlecombe, "UN Predicts 'Internet of Things'," July 6, 2009.

[9] D. Butler, "2020 Computing: Everything, Everywhere," Nature, vol. 440, no. 7083, pp. 402-409, 2006.

[10] S. Dodson, "The net shapes up to get physical," Guardian, 2008.

[11] N. Gershenfeld, R. Krikorian, and D. Cohen, "The Internet of Things,"

Scientific American, October, 2004.

[12] T. Al-Rousan, "Prospects of Cloud Computing In E-Government," International Journal of Advanced Computational Engineering and Networking. vol. 3, no. 10, 2015.

[13] R. Lombreglia, "The Internet of things," Boston Globe, pp. 76-83, 2005.

[14] A. Reinhardt, "A machine-to-machine Internet of things," 2004.

[15] G. Mark, and H. Haarstad, "Transparency and development: Ethical consumption and economic development through Web 2.0 and the Internet of things," Information Technologies \& International, 2011.

[16] B. Sterling, Shaping Things, Cambridge: Massachusetts Institute of Technology Press, 2005.

[17] G. Biamino, "Semantic model for socially aware objects, advances in Internet of things," vol. 2, pp. 47-55, 2012.

[18] S. Poslad, Ubiquitous Computing: Smart Devices, Environments and Interactions, John Wiley \& Sons, Ltd., p. 75, 2009.

[19] G. Kortuem, F. Kawsar, D. Fitton, and V. Sundramoorthy, "Smart objects as building blocks for the Internet of things," IEEE Internet Computing, vol. 14, no. 1, pp. 44-51, 2010.

[20] T. Al-Rousan and H. A Al Ese, "Impact of cloud computing on educational institutions: A case study," Recent Patents on Computer Science, vol. 8, no. 2, pp. 106-111, 2015.

[21] D. Singh, G. Tripathi, and A. J. Jara, "A survey of internet-ofthings: Future vision, architecture, challenges and services," in Internet of things (WF-IoT), 2014 IEEE world forum on. IEEE, 2014, pp. 287292.

[22] K. Habib, A. Torjusen, and W. Leister, "A novel authentication framework based on bio-metric and radio fingerprinting for the iot in ehealth," in Proceedings of International Conference on Smart Systems, Devices and Technologies (SMART), 2014, pp. 32-37. 
Int'I Journal of Computing, Communications \& Instrumentation Engg. (IJCCIE) Vol. 4, Issue 1 (2017) ISSN 2349-1469 EISSN 2349-1477

[23] D. D. Kumar and P. Venkateswarlu, "Secured Smart Healthcare Monitoring System Based On IoT," Imperial Journal of Interdisciplinary Research, vol. 2, no. 10, 2016.

[24] V. S. Gunge and P. S. Yalagi, "Smart Home Automation: A Literature Review," International Journal of Computer Applicatios, pp. 6-10, 2016. 